\title{
Context-specific outdoor time and physical activity among school-children across gender and age: using accelerometers and GPS to advance methods
}

\section{Charlotte Demant Klinker ${ }^{1}{ }^{*}$, Jasper Schipperijn ${ }^{1}$, Jacqueline Kerr $^{2}$, Annette Kjær Ersbøll $^{3}$ and Jens Troelsen ${ }^{1}$}

1 Department of Sports Science and Clinical Biomechanics, University of Southern Denmark, Odense, Denmark

${ }^{2}$ Department of Family and Preventive Medicine, University of California San Diego, San Diego, CA, USA

${ }^{3}$ The National Institute of Public Health, University of Southern Denmark, Copenhagen, Denmark

\section{Edited by:}

James Aaron Hipp, Washington

University in St. Louis, USA

Reviewed by:

Lisa C. McCormick, The University of Alabama at Birmingham, USA

Nicolas M. Oreskovic, Massachusetts General Hospital, USA

Jana A. Hirsch, University of

Michigan, USA

*Correspondence:

Charlotte Demant Klinker,

Department of Sports Science and

Clinical Biomechanics, University of

Southern Denmark, Campusvej 55,

Odense M 5230, Denmark

e-mail: ck@@sdu.dk
Introduction: Being outdoors has a positive influence on health among children. Evidence in this area is limited and many studies have used self-reported measures. Objective context-specific assessment of physical activity patterns and correlates, such as outdoor time, may progress this field.

Aims:To employ novel objective measures to assess age and gender differences in contextspecific outdoor weekday behavior patterns among school-children [outdoor time and outdoor moderate to vigorous physical activity (MVPA)] and to investigate associations between context-specific outdoor time and MVPA.

Methods: A total of 170 children had at least one weekday of $9 \mathrm{~h}$ combined accelerometer and global positioning system data and were included in the analyses. The data were processed using the personal activity and location measurement system (PALMS) and a purpose-built PostgreSQL database resulting in context-specific measures for outdoor time, outdoor MVPA, and overall daily MVPA. In addition, 4 domains (leisure, school, transport, and home) and 11 subdomains (e.g., urban green space and sports facilities) were created and assessed. Multilevel analyses provided results on age and gender differences and the association between outdoor time and MVPA.

Results: Girls compared to boys had fewer outdoor minutes $(p<0.05)$, spent a smaller proportion of their overall daily time outdoors $(p<0.05)$, had fewer outdoor MVPA minutes during the day $(p<0.001)$ and in 11 contexts. Children compared to adolescents had more outdoor minutes $(p<0.05)$. During school and within recess, children compared to adolescents had more outdoor MVPA $(p<0.001)$ and outdoor time $(p<0.001)$. A 1 -h increase in outdoor time was associated with 9.9 more minutes of MVPA $(p<0.001)$.

Conclusion: A new methodology to assess the context-specific outdoor time and physical activity patterns has been developed and can be expanded to other populations. Different context-specific patterns were found for gender and age, suggesting different strategies may be needed to promote physical activity.

Keywords: children, physical activity, accelerometer, GPS, spatial behavior, context-specific, outdoor behavior

\section{INTRODUCTION}

Being outdoors, as opposite to being indoors, may have a positive influence on a range of health parameters among children and adolescents (1). Being outdoors has also been identified as a correlate for more active play (2), enhanced physical activity levels (3-5), lower prevalence of overweight (6), and independent mobility (2). Being outdoors may help children and adolescents to reach 60 daily minutes of moderate to vigorous physical activity (MVPA); a minimum level recommended for children under the age of 18 by the World Health Organization and many national health authorities (7). Sustained low levels of physical activity are seen in many countries $(8,9)$, and often a decline in physical activity in the transition from childhood to adulthood is reported $(10,11)$.

Effective interventions or policies are needed to promote physical activity, and ecological models underpinning the importance of an active living lifestyle and built environmental influences, have received widespread recognition (12). The built environment consists of neighborhoods, roads, buildings, food sources, and recreational facilities: the places in which people live, work, are educated, eat, and play (13). If outdoor time is shown to be important for physical activity, policies to provide safe outdoor environments may be warranted. Evidence in this area is still 
limited and mixed as many studies on the association between the built environment and physical activity have used cross-sectional study designs and relied on self-reported data or daily averages from objective data. The association between the built environment and physical activity seems to be highly context-specific $(4,5)$, and inconsistencies in correlate studies may be partly explained by some studies measuring overall physical activity and not context-specific physical activity (e.g., physical activity during active transport, activity in urban green space or at playgrounds). An even better precision and perhaps correlation may be obtained if physical activity patterns are assessed (14-17) meaning that daily physical activity is assessed in different context throughout the day. Context-specific knowledge of physical activity patterns and correlates, such as outdoor time, may be a way to progress this field. Due to methodological challenges, this association has rarely been investigated using objective measures.

A valuable tool for improving the assessment of physical activity and outdoor behavior is the global positioning system (GPS). The GPS is a satellite-based global navigation system that provides a precise location at any point on the surface of Earth based on the position of satellites in the sky. The development of lightweight, affordable, and portable GPS receivers that can log individuals' locations continuously during consecutive days means that they can be used to objectively assess context-specific behavior. With the rapid development of the market for GPSs the battery life, memory capacity, and precision are improving (18). When combined with a device measuring physical activity, such as an accelerometer, it becomes possible to accurately assess patterns of context-specific physical activity (15-17, 19-24). The GPS receivers also collect information on the number of satellites used by and in view of the GPS receiver that can be used to provide estimates for outdoor times, and if assessed, outdoor physical activity (19, 20, 25, 26). A feasibility study by Tandon and colleagues among preschoolers concluded that it was feasible and valid to use a Qstarz GPS to distinguish indoor and outdoor time when using the personal activity and location measurement system (PALMS) (27) to process the data (26). Furthermore, information on speed and distance traveled can be used to assess mode of transport (28, 29). At the moment, new evidence about context-specific physical activity behaviors is being generated on the basis of combined accelerometer and GPS data and this paper is part of a developing research area.

The aim of this paper is to employ objective measures to assess the context-specific outdoor weekday pattern among schoolchildren and determine which contexts contribute to most outdoor time. Furthermore, the aim is to assess the contexts where weekday outdoor MVPA occurs and investigate how much of total daily MVPA is outdoors. As gender is a strong correlate for physical activity, and age a probable correlate (3), age and gender differences were assessed for these two aims. Finally, the aim was to investigate the association between context-specific outdoor time and MVPA.

\section{MATERIALS AND METHODS \\ PARTICIPANTS AND PROCEDURES}

Children in grade 5-8 (11-16 years old) were recruited from four schools, participating in the When Cities Move Children (WCMC) study. The WCMC study is a natural experiment conducted in a deprived neighborhood in Copenhagen, the capital of Denmark, evaluating how major improvements to the built environment influences physical activity and movement patterns. There are 9300 people living in the district; children comprise $20 \%$ of the population, and almost $70 \%$ of the children are immigrants or descendants of immigrants (30). Only baseline data collected in 2010-2011 were employed for the current analyses conducted in summer 2013. We chose to sample participants in a time period where it was hypothesized that the average day length, temperature, and rain were comparable in a Danish context. Data from three schools were collected in spring while data from the fourth school were collected in fall, corresponding to $85 \%$ of the data collected in spring and $15 \%$ in fall. There were no differences between participants from these two seasons by gender, age, BMI, MVPA or combined accelerometer, and GPS wear time.

Eligible children $(N=623)$ and their parents received personalized information about the nature and procedures of the study in Danish and if needed in one of four other languages (Arabic, Somali, Turkey, and Urdu) to match the ethnic background of the parents. The parents and children were notified that participation was voluntary and that they could withdraw at any stage. A passive informed consent procedure was used, where students were included unless the parents withdrew consent as this procedure has been found to be ethically appropriate in low-risk research in adolescents (31). The Danish Ethical Regional Committee reviewed the study protocol and concluded that formal ethics approval was not required. The study is registered and approved by the Danish Data Protection Agency (reference number: 2009-41-3943). Consent was obtained from 523 children and there were no overall differences between responders and non-responders by gender, ethnicity, BMI, or parental work status. However, the non-response was unequally distributed by age and school with the drop-out being greater among adolescents (children $11.7 \%$, adolescents $21.4 \%, p=0.001)$ and in two schools $(p<0.001)$.

The inclusion criteria were at least one valid weekday of $9 \mathrm{~h}$ combined accelerometer and GPS wear time, excluding day 1 data, weekend data, participants not staying in their primary home during data collection (for participants with divorced parents), and participants who did not have any outdoor data. Data from day 1 were removed as the equipment was fitted at different times during the day, leaving the participants with unequal opportunities to obtain enough hours to become a valid day. Furthermore, the analyses conducted required a full day behavior pattern. Weekend data were removed as weekday data provided the greatest variability in domains and subdomains and because weekends and weekdays are not directly comparable in terms of domains/subdomains, i.e., children do not attend school on weekends. Data from children with divorced parents who stayed in their secondary home during data collection were removed as only the primary parent's address was known to be located within the assessed neighborhood. Due to a software problem with the initializing system used to set-up the GPS devices, almost all GPS on two schools did not record the satellite signal to noise ratio (SNR), which meant that the time spent outdoors could not be assessed. The majority of participants from these two schools were excluded, leaving 204 participants with combined accelerometer and GPS data and outdoor time measures. Eight participants were excluded as they only 
had weekend data or data from day 1, 6 participants were excluded as they only were in their secondary home during data collection, and 20 were excluded as they did not have one valid day of $9 \mathrm{~h}$ of combined weekday data. This led to a total sample for this study of 170 participants out of the 523 consenters (32.5\%). There were no differences in background characteristics, such as gender, BMI, parental employment status, and immigrant status, between those who provided complete measures $(n=170)$ and those who were excluded ( $n=353)$. However, the drop-out was unequally distributed by school $(p<0.001)$ and age $(p<0.001)$. Figure 1 displays a flow diagram of the reduction of the population.

\section{PHYSICAL ACTIVITY - ACCELEROMETER MEASURES}

Objective physical activity levels were assessed using the tri-axial Actigraph GT3X accelerometer during seven consecutive days. On day 8 , the participants handed in the equipment. Only the vertical axis was used for this study. The accelerometer has the ability to yield measures of volume, frequency, intensity, and duration of children's physical activity (32). Several reviews have concluded that accelerometers provide an accurate, reliable, and practical objective measure of physical activity in children and adolescents $(33,34)$. The data were recorded at $2 \mathrm{~s}$ epochs. All students were instructed to wear the monitor on their hip during waking hours and to take it off only for showering, bathing, or any water sports. They were asked to record in a diary the times they took the monitor off and the reason for doing so. Data from the returned monitors were downloaded using the ActiLife 4.4.1 software and screened for file size to detect potential equipment or download problems.

\section{CONTEXTS - GPS MEASURES}

QStarz BT-Q1000X GPS units were used to record movement. The QStarz unit has shown relatively high accuracy across a range of sites (e.g., canopy and open sky) and good inter-unit reliability compared to other units $(18,29,35)$. The GPS units were set-up using BT747 open source software (bt747.org). Units were configured to log data every $15 \mathrm{~s}$, a compromise between optimal frequency (i.e., $2 \mathrm{~s}$ as the accelerometer) and data storage capacity of the units over a 7-day period. The units were set to stop logging when the memory was full and to record: date, time, longitude and latitude (used to calculate location), elevation, speed (used to calculate transport mode), and the number of satellites in view and used (used to calculate the Signal-to-Noise-Ratio (SNR)). SNR's can be used to estimate if the GPS is outdoors. After set-up, the fully charged GPS were turned off. On day 1 of data collection the research team turned the GPS units on, and taped the on/off button to prevent it from sliding to off. The children were instructed to leave the tape on, and not turn the unit off during data collection. They were instructed to wear the GPS on the same belt as the accelerometer but on the opposite hip, and only to take it off as instructed with the accelerometer. After data collection, the data were downloaded using bt747 software and screened for file size to detect the potential equipment or download problems.

Participants who lost or had malfunctioning devices during data collection, and informed the research team, had their device exchanged, and their data were later merged into one data-file.

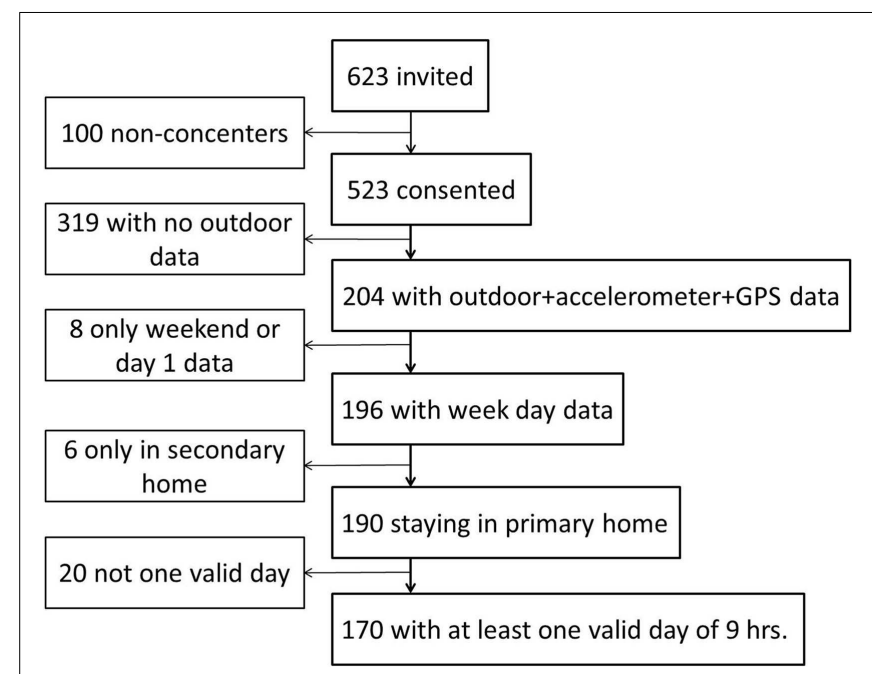

FIGURE 1 | Reduction of the study population

A total of 14 participants lost one or both devices, or returned malfunctioning devices when data collection was complete.

\section{CORRELATES}

Children's immigrant status was obtained from Statistics Denmark using a unique personal identification number assigned to all people in Denmark (36) and children were categorized as Danish versus immigrant (not born in Denmark) or descendent (born in Denmark but parents not born in Denmark). Parental employment status was obtained from Danish registers on personal labor market affiliation (37) and categorized as parents working versus one or both unemployed. Age was dichotomized into grade 5-6 (age 11-13) versus grade 7-8 (13-16), to best approximate children and adolescents. Information on self-reported height and weight was obtained from a questionnaire (E-survey) completed during data collection. BMI was calculated using Cole's age and gender specific cut off points (38) and included as a continuous variable.

\section{STEPS TAKING TO INCREASE VALIDITY}

Questionnaire data were used to assess if children living with divorced parents were residing in their primary home (i.e., the local neighborhood) during the data collection period. Participants completed a daily diary during data collection to assess non-wear and changes to the ordinary school schedule. Students were asked to note school (non-)attendance times, and if it had been a regular school day (if not, why not?). The schools furthermore provided detailed class timetables for the data collection period including information on start and end of school days, recess and physical education (PE). These measures were used to adjust and improve the quality of the combined GPS and accelerometer data during data processing.

\section{DATA PROCESSING}

The PALMS is a web-based application capable of combining activity data (e.g., accelerometers) with location data (GPS). PALMS aggregated and processed the accelerometer data to provide values for MVPA using $15 \mathrm{~s}$ cut points $(39,40)$. Evenson cut 
points (39) were used in this study with 574 counts per $15 \mathrm{~s}$ as threshold for MVPA. Continuous periods of $60 \mathrm{~min}$ of zero values were classified as non-wear time and removed $(33,41)$. PALMS processed the GPS data by identifying invalid data points using extreme speed or extreme changes in distance and elevation, and replaced invalid points by imputing data from the last known valid point, for up to $10 \mathrm{~min}$. Using algorithms that utilize SNR PALMS categorized epochs as occurring indoors or outdoors. For this study, PALMS marked the locations as outdoor when the total SNR of all satellites in view exceeded a threshold of $250(26,29)$. Furthermore, PALMS identified and categorized trips (defined as a continuous period of movement of at least $3 \mathrm{~min}$, allowing for stationary periods of maximum $5 \mathrm{~min}$ ) into three modes: walking, bicycling, and vehicle (29). Processed GPS data were then matched to the accelerometer data in $15 \mathrm{~s}$ epochs, forming a PALMS dataset. The PALMS dataset consisted of $15 \mathrm{~s}$ accelerometer epochs with the following information appended: location (GPS coordinates: latitude and longitude), activity intensity (MVPA), outdoor (yes, no), and trip mode (walk, bicycle, and vehicle). In case no GPS signal was available the accelerometer epochs were retained to calculate the daily physical activity variables. The PALMS dataset is very rich in information and no available data management systems were able to handle the data load, or the specific requirements developed to obtain high quality, precise context-specific measures. Therefore, a purpose-built PostgreSQL database was developed. The PostgreSQL database was set-up to combine PALMS datasets with data from participant dairies, class timetables, and location data from a Geographical Information System (GIS, ArcGIS 10.1) to compute variables for days and context-specific settings. The context-specific settings included were the active living domains: leisure, school (scheduled school hours) (42), transport, and home (43) and a range of subdomains within these domains reflecting places where children and adolescents can be involved in MVPA. The subdomains constituting leisure were: school grounds (outside scheduled school hours) (44), clubs (after school programs), sports facilities (45), playgrounds (46, 47), urban green space $(19,20)$, shopping centers, and "other places." The Municipality of Copenhagen provided the addresses of public schools, clubs, sports facilities, and playgrounds, enabling a manual digitizing of school grounds, clubs, sports facilities, and playgrounds in GIS. All urban green spaces were available from the Danish Geodata Agency. Major indoor shopping centers were identified online, and manually digitalized in GIS. Epochs not categorized as school, home, transport, or other leisure subdomains were categorized as other places. Epochs were assigned to school, recess, or PE according to the school schedule, adjusting for individual variations based on the individual student diary, and total recess and $\mathrm{PE}$ were then assessed within the school domain. All epochs classified by PALMS as trips, and not part of any other domain, constituted the transport domain, and PALMS trips were dichotomized into active (walking and biking) and passive (vehicle) transport. All students' primary addresses were geocoded and each home was digitalized manually in GIS to constitute the home domain. A house was in GIS defined as the parcel, while an apartment was defined as the building and adjacent outdoor area. No subdomains were defined within the home domain. A 10-m Euclidian buffer was applied to all GIS derived domains and subdomains to account for signal and location errors. The database applied a hierarchical process to ensure an epoch could only be assigned to one context. All epochs belonging to the school domain were categorized first, followed by epochs belonging to first the home and then the leisure domain. Epochs belonging to a trip were then assigned to the transport domain, while the left over epochs finally were assigned to the leisure subdomain "other places". The data were aggregated on an individual level by day, domain, and subdomain in the database before being imported into a statistical software package for further analyses.

\section{OUTCOME MEASURES}

For weekdays, 4 domains, and 11 subdomains, five daily contextspecific outcome measures were calculated and used in this study: minutes of outdoor time, the proportion of time spent outdoors, minutes of outdoor MVPA, the proportion of MVPA spent outdoor, and MVPA. Proportion of time spent outdoors was calculated as minutes of outdoor time out of (wear) time during the average weekday or context-specific setting and hence expresses the proportion of time accumulated in a context that was outdoors. The proportion of MVPA spent outdoors was calculated as minutes of outdoor MVPA in a weekday, domain or subdomain out of total minutes of MVPA accumulated in the day, domain or subdomain and hence expresses the percentage of how much of all MVPA accumulated during the day, domain, and subdomain that is occurring outdoors. These measures were included to account for potential differences in movement patterns among groups, e.g., boys and girls may spend equal amount of time in a context but one part may spend double the amount of outdoor time.

\section{DATA ANALYSES}

All analyses were performed using STATA SE12. Descriptive statistics were used to assess age, gender, and BMI by means of frequency distribution (\%), and mean and standard deviation. Median and inter quartile ranges (IQR) were used to describe minutes of daily MVPA and wear time as these variables were not normally distributed. Univariable analyses were performed to evaluate the association between the two age groups and between boys and girls using a chi-square, $t$-test, or Wilcoxon rank-sum test. As the majority of outcome measures were not normally distributed, median and IQR were used to describe four of the outcome measures: outdoor times, the proportion of time spent outdoors, outdoor MVPA and the proportion of MVPA spent outdoors in total, domains and subdomains by gender, age, and totals. Multilevel analyses were used to provide results on age and gender differences. All models included students within school, further adjusting for BMI, number of valid weekdays (1-4) and daily wear time (models based on total days) or time in overall domain being investigated, e.g., the subdomain playground was adjusted for time in the leisure domain. Each model accounted for the nested nature of children within schools (48) by including school as a fixed effect. Models with a non-normal distribution of the residuals (49 out of 64 models) had their outcome transformed to fulfill the model assumptions, 22 by square root, 17 by $\log , 8$ by $x^{2}$, and 2 by $x^{3}$ transformation. For the $17 \log$ transformed models, zeros were replaced with a small number $(0.03125$ corresponding to half the value of the lowest non-zero number across the models) before 
transformation. The transformed model $p$-value and the untransformed model coefficient for age and gender differences are shown for ease of interpretation. Age and gender interactions were found in 15 out of 64 models ( ignificance level $p=0.05$ ). The interaction $p$-value and the significant gender and age subgroup differences are presented separately. Multilevel analyses were used to provide unadjusted and adjusted results on the association between MVPA and outdoor time during the total day, domains, and subdomains. All the adjusted models accounted for the nested nature of children within schools by including school as a fixed effect, further adjusting for age, gender, BMI, number of valid weekdays (1-4) and registered time in day (models based on total days), or time in overall domain being investigated. All models were tested for interactions between outdoor time, age, and/or gender (significance level $p=0.05$ ) to investigate if the association was persistent across age and gender groups. An interaction was present in 7 out of 16 contexts; however, the pattern of the association (i.e., the size of the $p$-value) was the same across all subgroups with four exceptions. For ease of interpretation, the results from the untransformed models showing totals rather than subgroups are shown, while the text specifies the four subgroup exceptions.

\section{RESULTS}

\section{PARTICIPANTS}

Table 1 shows characteristic for the study participants $(n=170)$. On average, the participants had a daily median of $12.9 \mathrm{~h}$ (IQR, 11.7-13.6) of combined accelerometer and GPS data and a mean of 2.7 valid days (SD 1.1) out of 4 possible. Boys compared to girls had more minutes of daily MVPA (82.8 versus $61.2 \mathrm{~min}, p<0.001$ ). As expected, BMI was greater among adolescents compared to children $(p<0.05)$.

\section{TIME OUTDOOR PATTERN}

Table 2 shows the daily median minutes of outdoor time (minutes, IQR) in total, domains, and subdomains by gender and age, and age and gender differences assessed in multilevel analyses. Across all groups, the majority of outdoor time was accumulated during the school hours, followed by leisure time, transport, and home. However, the pattern was less clear among adolescents.

While boys were outdoors $226.7 \mathrm{~min}$ per day, girls were outdoors $194.5 \mathrm{~min}$ per day $(p<0.05)$ (Table 2$)$. This difference was predominantly due to a difference during leisure, where boys were outdoors $71.9 \mathrm{~min}$ and girls were outdoors only $45.0 \mathrm{~min}$ $(p<0.001)$. Within leisure, boys spent more time outdoors when in sports facilities and other places (all $p<0.05$ ). Boys also spent more time outdoors when in transport $(p<0.05)$. There was no gender difference in the time spent outdoors during school or home.

Children were outdoors a median of $226.5 \mathrm{~min}$ per day and adolescents $172.6 \mathrm{~min}$ per day $(p<0.05)$ (Table 2$)$. This difference originated predominantly from a difference during school hours, were children compared to adolescents had almost the double amount of outdoor time (96.5 versus $44.5 \mathrm{~min}, p<0.001$ ). Children also spent more time outdoors when at school grounds outside school hours ( 15.3 versus $4.1 \mathrm{~min}, p<0.05$ ) but spent fewer minutes outdoors than adolescence during transport $(24.8$ versus $30.2 \mathrm{~min}, p<0.05)$.

Table 3 shows the daily median proportion of time spent outdoors (\%, IQR) in total, domains, and subdomains by gender and age, and age and gender differences assessed in multilevel analyses. Boys spent a greater proportion of their time during a day being outdoors: boys were outdoors $29.0 \%$ of the day while girls were outdoors $22.2 \%$ of the day $(p<0.05)$. The proportion of time spent outdoors when in a particular domain or subdomain varied; with home being the place where the lowest proportion of time was spent outdoors (girls $8.5 \%$, boys $7.9 \%$ ) and playgrounds had the highest proportion (girls $87.5 \%$, boys $99.8 \%$ ). Besides spending a larger proportion of time outdoors in leisure, sports facilities, other places, and in transport, boys compared to girls also spent a greater proportion of their time outdoors in school grounds, during recess, and PE. No gender difference was detected in the proportion of time spent outdoors when in active transport, despite boys spending significant more minutes outdoors in active transport.

A trend of an overall age difference in the proportion of time spent outdoors during the total day was detected with children being outdoors $27.3 \%$ of the day and adolescents $20.6 \%$ of the day $(p=0.05)$ (Table 3). Children compared to adolescents accumulated a larger proportion of their MVPA at school grounds $(p<0.05)$, during school hours $(p<0.001)$, and during recess $(p<0.001)$.

An analysis of age and gender interactions further revealed that adolescent girls had less daily outdoor time and spent a lower proportion of time outdoor on school grounds and at other places

Table 1 | Study participants $(n=170)$.

\begin{tabular}{|c|c|c|c|c|c|}
\hline & Girls & Boys & Children & Adolescents & Total \\
\hline Population (\%) & $87(51.2)$ & $83(48.8)$ & $129(75.9)$ & $41(24.1)$ & $170(100)$ \\
\hline Mean age (SD) & $12.9(1.2)$ & $12.8(1.0)$ & $12.4(0.7)^{* * *}$ & $14.2(0.8) * * *$ & $12.8(1.1)$ \\
\hline Mean valid days (SD) & $2.7(1.1)$ & $2.6(1.1)$ & $2.7(1.1)$ & $2.4(0.9)$ & $2.7(1.1)$ \\
\hline Median daily minutes MVPA (IQR) & $61.2(46.7-75.8) * * *$ & $82.8(58.4-99.1) * * *$ & $69.5(53.5-91.9)$ & $58.3(46.8-85.6)$ & $68.4(52.0-91.8)$ \\
\hline
\end{tabular}

${ }^{*}$ Significant difference $p<0.05$.

***Significant difference $p<0.001$.

${ }^{a} n=156$.

$B M I$, body mass index; IQR, inter quartile range; MVPA, moderate to vigorous physical activity; SD, standard deviation. 


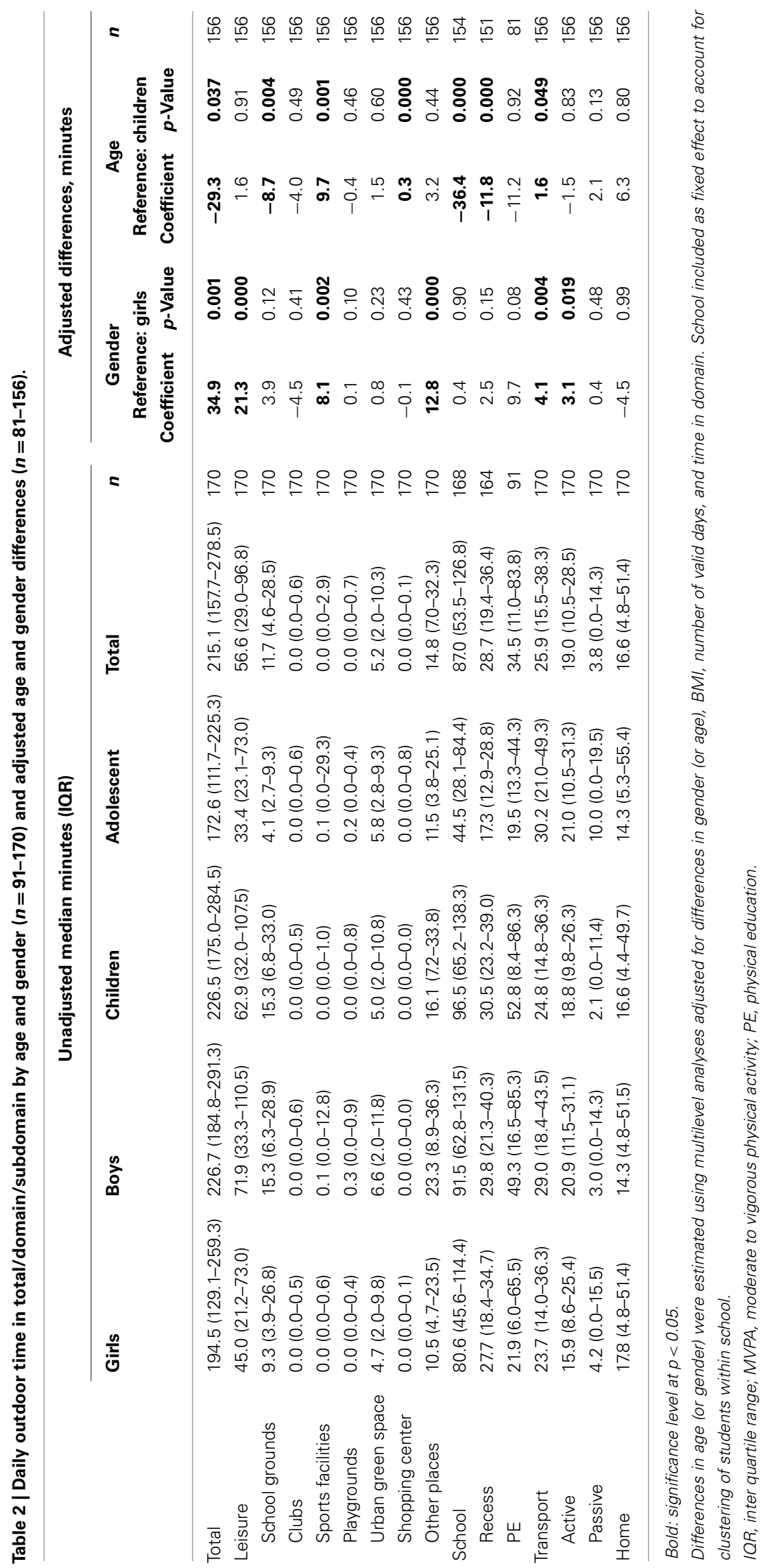




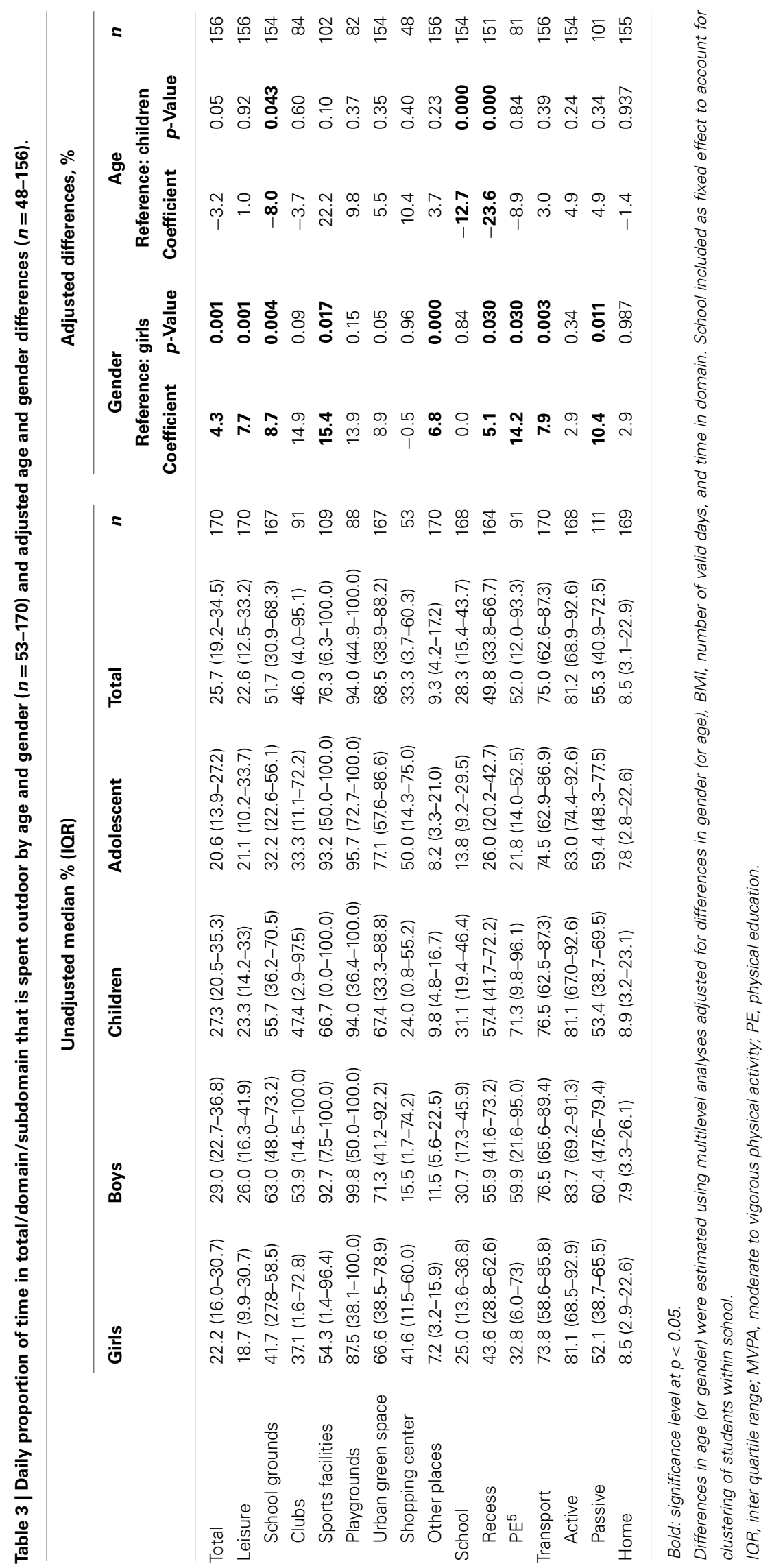


than child girls and boys (Table 6). Girl children also spent a smaller proportion of time in other places than boys. Adolescent boys spent more leisure time outdoor and spent a larger proportion of time outdoor than girls and child boys. Child boys spent more leisure time outdoor and spent a larger proportion of outdoor time than adolescent girls. Adolescent boys spent more time outdoors in urban green space than adolescent girls.

\section{OUTDOOR MVPA PATTERN}

Table 4 shows the daily median minutes of outdoor MVPA time (outdoor MVPA minutes, IQR) in total, domains, and subdomains by gender and age and age and gender differences assessed in multilevel analyses. Girls accumulated a daily median of $42.3 \mathrm{~min}$ of outdoor MVPA, while boys accumulated a daily median of 61.8 min of outdoor MVPA $(p<0.001)$. In 10 out of the 15 investigated contexts, boys compared to girls accumulated more minutes of outdoor MVPA, with no gender differences present in clubs, playgrounds, urban green space, shopping centers, and passive transport $(p>0.05)$.

Among children and adolescents, no overall difference was found in how many minutes of outdoor MVPA were accumulated during the whole day $(p>0.1)$ (Table 4). Children compared to adolescents had more outdoor MVPA during school hours and recess $(p<0.001)$ while adolescent compared to children had more outdoor MVPA at sport facilities, shopping centers, and passive transport $(p<0.05)$.

In the analyses investigating the proportion of MVPA occurring outdoors during the day and in different contexts (Table 5), a significant gender difference was detected overall and in 6 out of the 15 investigated contexts. Boys accumulating a larger proportion of their MVPA outdoors when in leisure overall, in school grounds, sports facilities, playgrounds, school, and PE. During the total day, $73.8 \%$ of boys MVPA was spent outdoors with girls spending $65.3 \%$ of their MVPA outdoors $(p<0.001)$. No overall difference was found between children and adolescents in the proportion of daily MVPA that was spent outdoors, but children spent a larger proportion of their MVPA outdoors during school hours and recess $(p<0.001)$.

When in transport, clubs, sport facilities, playgrounds, urban green space, and in recess a high proportion of MVPA took place outdoors for both boys and girls, and children and adolescents (84.8-100\%). Boys and children also accumulated a large proportion of their MVPA outdoors when in PE (boys 85.3\%, children 91.9\%) (Table 5).

Adolescent boys accumulated more outdoor MVPA minutes in urban green space and home than child boys and girls (Table 6). Adolescent boys also spent a larger proportion of their MVPA outdoors during leisure, in school grounds, and other places. Adolescent boys spent a larger proportion of their MVPA outdoors when at home compared to adolescent girls. Child boys had more minutes of outdoor MVPA compared to adolescent boys and girls. Child girls spent a lower proportion of their MVPA outdoors while at sports facilities compared to adolescent girls and boys.

\section{TIME OUTDOOR AND MVPA}

In multilevel analyses, time spent outdoors (hours) was a significant predictor of MVPA (minutes) both in unadjusted models and in models adjusted for potential confounders (gender, age, BMI, number of valid days, time in day, or overall domain) (Table 7). Models were run for days, domains, and subdomains to investigate if the association varied by context, but a consistent relationship was found throughout the day (all $p<0.001$ ) with only four exceptions detected in supplementary analyses investigating interactions between outdoor time, gender, and age (data not shown). No association was found between outdoor time and MVPA for child boys when at shopping centers $(p>0.1)$ and in transport $(p>0.1)$. Also a weaker association was found for adolescent girls when in transport $(p=0.05)$ or at home $(p=0.06)$. During the course of the whole day, a 1-h increase in outdoor time was associated with 9.9 more minutes of MVPA. An association was also found for contexts in leisure time where a 1 -h increase in outdoor time was associated with an increase of 23.5 more minutes of MVPA in school grounds, 20.2 more minutes of MVPA in urban green space, and 18.6 more minutes of MVPA when at sports facilities. One more hour of outdoor time during active transport was associated with 28.5 more minutes of MVPA (all $p<0.000$ ).

\section{DISCUSSION}

This study investigated the volume and pattern of context-specific weekday outdoor time, outdoor MVPA, and the association between context-specific daily MVPA and outdoor time using combined accelerometer and GPS data for 170 children aged 1116 years old. Four domains, 11 subdomains, and daily medians were assessed as context-specific measures and age and gender differences were investigated. A different pattern was found for boys and girls, as well as for children and adolescents. Girls compared to boys had fewer outdoors minutes and spent a lower proportion of their daily time outdoors overall and in the majority of investigated contexts. Girls compared to boys had fewer outdoor MVPA minutes during the day and in 11 contexts. A less consistent difference was found for the proportion of MVPA spent outdoors; gender differences were only detected in five contexts. During the total weekday, children compared to adolescents had more outdoor minutes $(p<0.05)$ while no difference in daily outdoor MVPA behavior was found. However, across all investigated outcomes a difference in behavior in the school context was detected, with children engaging in more outdoor MVPA and spending more time outdoors during school hours and within recess. Finally, it was found that outdoor time was a correlate for MVPA across the total day, all domains and subdomains.

Overall, 21.8-29.3\% of time was spent outdoors, corresponding to approximately $3 \mathrm{~h}$ a day. Compared to other studies, even though the studies are not directly comparable as the methods used differ, it appears that the Danish children studied were spending more time outdoors than children included in studies from the UK $(19,25)$, Australia $(2,6)$, and Switzerland (49). This discrepancy could be due to outdoor time being measured differently; the Australian and Swiss studies relied on self-report data and the UK studies used a GPS device that assessed outdoor time differently from the present study. Another difference might be time of year when the data were collected as seasonality and weather conditions previously have been related to objectively assessed physical activity in children (50) and this association is likely to also apply to outdoor times. 


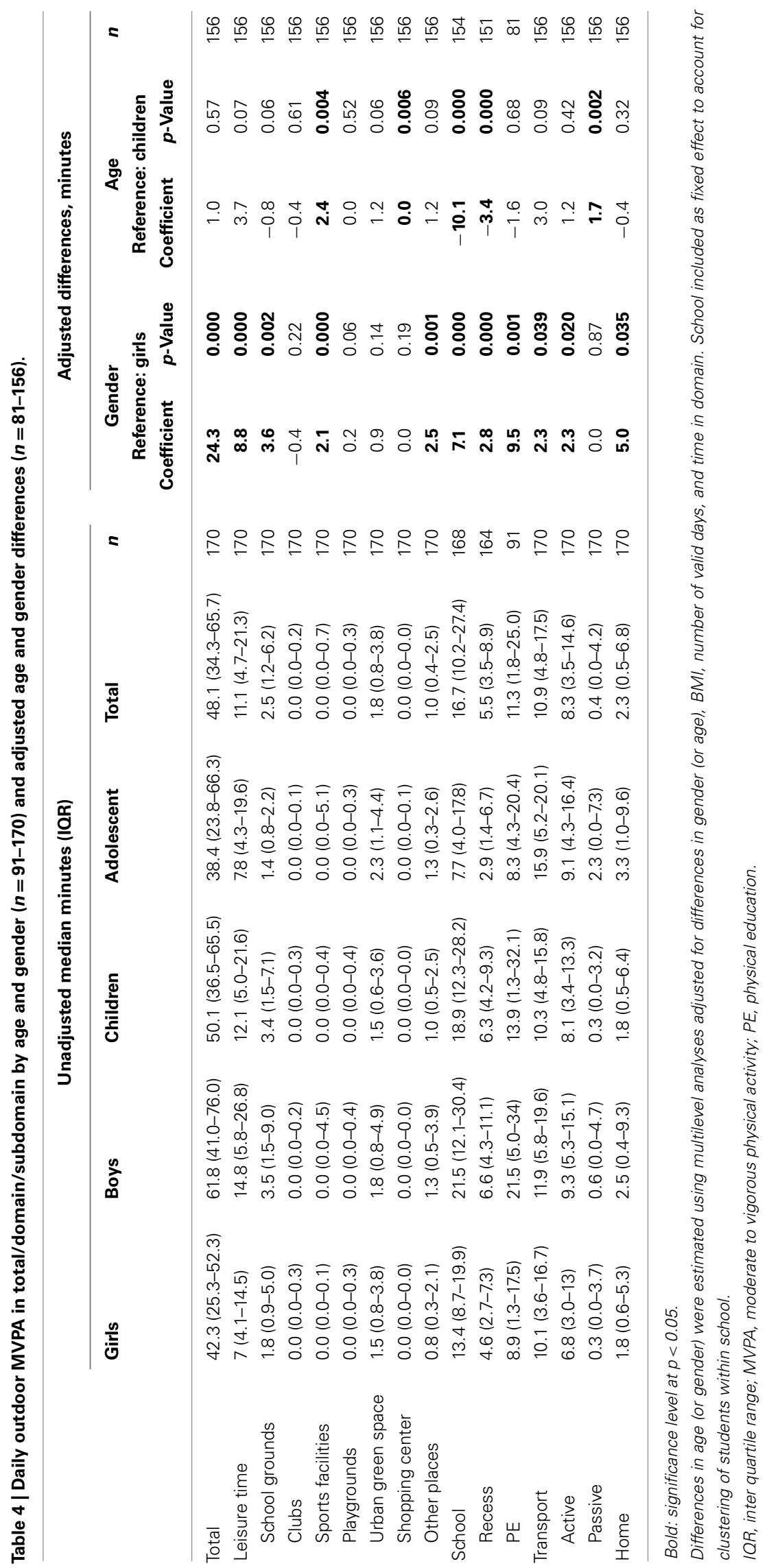




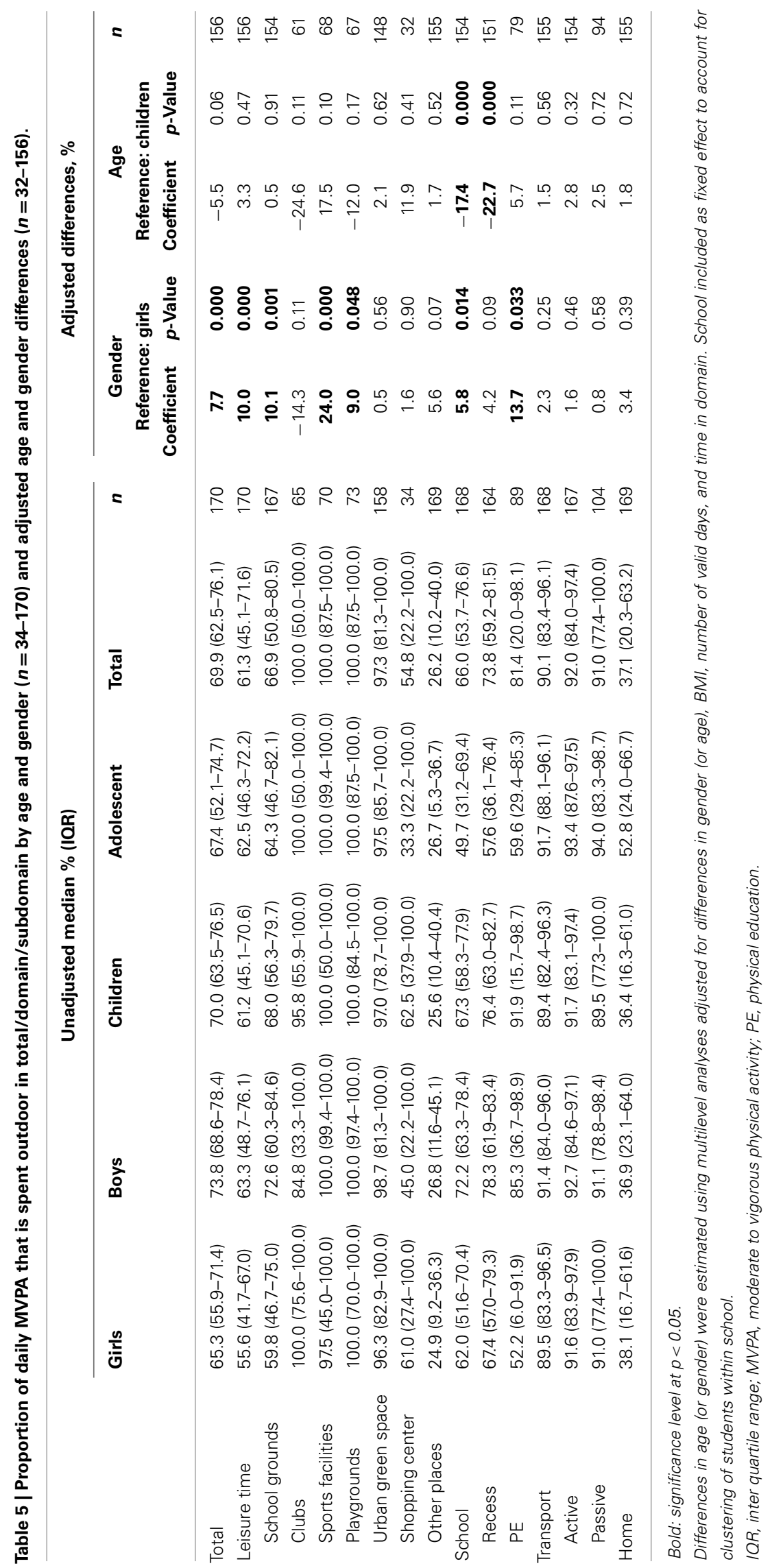




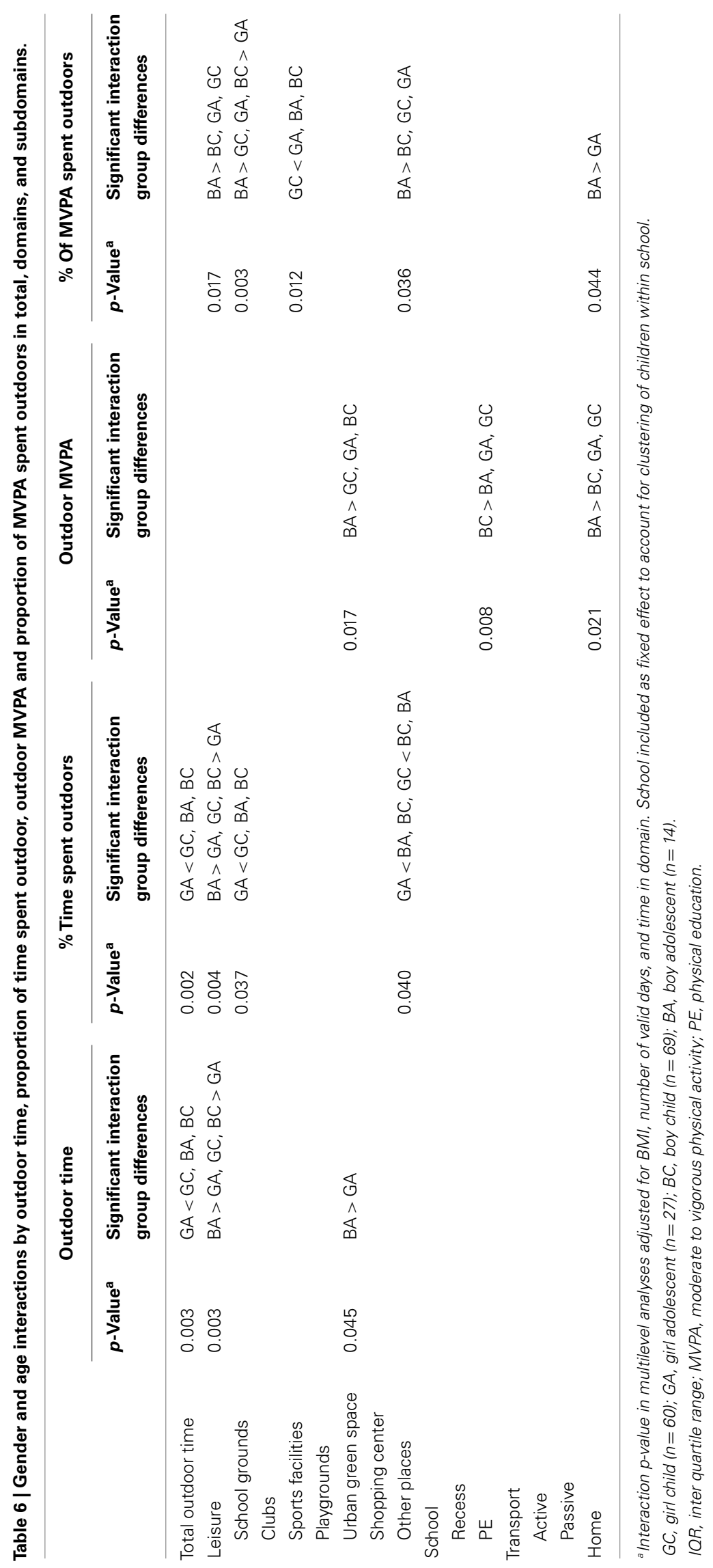


Table 7 | Association between time outdoors (hours) and MVPA (minutes) in total weekdays, domains, and subdomains.

\begin{tabular}{|c|c|c|c|c|c|c|}
\hline & \multicolumn{3}{|c|}{ Model 1} & \multicolumn{3}{|c|}{ Model 2} \\
\hline & Coef. & $p$ & $95 \% \mathrm{Cl}$ & Coef. & $\boldsymbol{p}$ & $95 \% \mathrm{Cl}$ \\
\hline Total day & 10.9 & $<0.001$ & $8.0-13.8$ & 9.8 & $<0.001$ & $6.9-12.8$ \\
\hline Leisure time & 14.8 & $<0.001$ & $12.3-17.4$ & 13.5 & $<0.001$ & $10.7-16.4$ \\
\hline School grounds & 21.7 & $<0.001$ & $19.5-23.9$ & 23.5 & $<0.001$ & $21.1-25.9$ \\
\hline Clubs & 10.7 & $<0.001$ & $9.0-12.4$ & 11.2 & $<0.001$ & $9.4-13.0$ \\
\hline Sports facilities & 18.0 & $<0.001$ & 16.4-19.6 & 18.6 & $<0.001$ & $16.8-20.3$ \\
\hline Playgrounds & 16.3 & $<0.001$ & 15.5-17.1 & 16.4 & $<0.001$ & $15.6-17.3$ \\
\hline Urban green space & 20.2 & $<0.001$ & $18.5-21.8$ & 20.2 & $<0.001$ & $18.4-21.9$ \\
\hline Shopping center & 15.9 & $<0.001$ & $12.5-19.3$ & 17.3 & $<0.001$ & $13.7-20.9$ \\
\hline Other places & 7.3 & $<0.001$ & $4.9-9.7$ & 5.5 & $<0.001$ & $2.9-8.1$ \\
\hline School & 9.0 & $<0.001$ & $6.5-11.4$ & 8.3 & $<0.001$ & $5.8-10.8$ \\
\hline Recess & 11.6 & $<0.001$ & $7.8-15.4$ & 8.5 & $<0.001$ & $4.3-12.6$ \\
\hline $\mathrm{PE}$ & 13.1 & $<0.001$ & $8.2-18.0$ & 10.9 & $<0.001$ & $5.9-15.9$ \\
\hline Transport & 21.2 & $<0.001$ & $17.7-24.6$ & 15.0 & 0.002 & $5.6-24.4$ \\
\hline Active & 29.3 & $<0.001$ & $25.4-33.2$ & 28.5 & $<0.001$ & $23.5-33.6$ \\
\hline Passive & 10.6 & $<0.001$ & $8.6-12.6$ & 6.5 & $<0.001$ & $3.6-9.3$ \\
\hline Home & 10.1 & $<0.001$ & 8.3-11.9 & 9.1 & $<0.001$ & $7.1-11.2$ \\
\hline
\end{tabular}

Model 1: unadjusted multilevel analyses.

Model 2: multilevel analyses adjusted for age, gender, BMI, valid days, time in day/domain, and clustering of students within schools.

Coef., mean increase in minutes of MVPA associated with a 1-h increase in outdoor time.

IQR, inter quartile range; MVPA, moderate to vigorous physical activity; PE, physical education.

In agreement with other studies, boys spent more time outdoors than girls $(6,25)$ and children spent more time outdoors compared to adolescents (49). The majority of outdoor time was for all groups occurring during school hours followed by leisure time. This study is one of the first to estimate the proportion of outdoor time occurring in domains during the day and further studies are needed to confirm this finding.

Between 62.3 and $71.0 \%$ of all total daily MVPA was accumulated outdoors. In the PEACH study, it was found that $26.4-35 \%$ of MVPA took place outdoors on weekdays outside the school hours (19). Even though not directly comparable, it seems like the present study population experienced a greater proportion of MVPA outdoors. Similar to the PEACH study, outdoor time in the present study was found to be a significant predictor of daily MVPA in all investigated contexts, and a 1-h increase in outdoor time was associated with almost 10 more minutes of MVPA per day. This study shows a stronger relationship between outdoor time and MVPA than a previous study using self-reported measures from parents (6). Here it was found that for every additional hour spent outdoors per week, MVPA increased by $27 \mathrm{~min}$ per week (almost 4 min per day) among 10-12 year old children. The stronger association found in this study may be due to self-reported measures from children or parents being imprecise, and the inclusion of GPS data may provide a more reliable estimate of actual outdoor time, and the accelerometer a more reliable estimate of physical activity. The overall level of daily MVPA among the participants in our study was also high, reaching a daily median of $67.3 \mathrm{~min}$ of MVPA. As this study is based on a cross-sectional sample it is not possible to conclude the causal direction of outdoor time and MVPA. Longitudinal studies are needed to establish a causal relationship between outdoor time and MVPA, but promoting outdoor time among the investigated group may have a range of health benefits beyond the association with physical activity. Modifiable characteristics in the neighborhood such as sidewalks, parallel or grouped parking places, traffic safety, and roundabouts have been associated with outdoor play (51), and parental concern about traffic safety has been associated with less time playing outdoors (49, 51). When planning urban renewal programs or new neighborhoods, factors that may be associated with increased or decreased outdoor time are important to consider.

No studies to date have investigated what is needed to obtain reliable estimates of children's context-specific physical activity patterns based on the combination of accelerometers and GPS, and measurement decisions are relaying on recommendations for accelerometer studies. Future methodological studies are needed to investigate if and how the use of combined accelerometers and GPS should differ in the design from a study based solely on accelerometers. Experiences from this study indicate some specific areas that need careful consideration before embarking on research studies using combined accelerometer and GPS measurements. A larger drop-out was seen in this study compared to a similar project using only accelerometers conducted simultaneous (52). Both during data collection (fewer persons consented, more opted out or lost equipment during data collection, device-failure, e.g., software resulting in missing information on SNR), but also in the data analyses were it was evident that less rigid demands had to be applied to wear time and number of valid days to retain a reasonable study population. It may be reasonable to hypothesize that the inclusion of the GPS placed a greater burden on the participants, leading to less compliance with the study protocol. 
Therefore, it is recommended that studies using accelerometer and GPS should consider: (a) to oversample or (b) to ask participants to wear the equipment for a longer period of time. Solution: (a) implies either a longer data collection period or a greater pool of equipment while solution (b) again increases the participant burden. A method seen and recommended in accelerometer studies to increase the number of valid participants is to quickly download and screen data to see if a participant complied with the protocol. If not, they are asked to re-wear the accelerometer (53). Similar procedures for the GPS may also be feasible; however, it is time-consuming.

Using a combination of accelerometers and GPS to develop context-specific measures has a large potential to lead to new knowledge on physical activity, inform the development of new interventions, and perhaps later lead to new policies or recommendations for specific subgroups. Still, it is not an easy fix. The data collection is more complex, the recruitment of participants harder, and the data processing and complexity of the data is overwhelming. Practical issues of data storage capacity and run times of 2-3 weeks to generate variables in the purpose-built database for subsamples of 100 participants were a reality in this study, making the analyses time-consuming and labor-intensive. The development of PALMS is one major step toward a resource that can help researchers to start process their data. Integrating the methodology behind this study into PALMS has the potential to increase the number of studies investigating context-specific behaviors, as well as lead to greater conformity across studies, making comparisons possible, and hence perhaps increase knowledge on generalizable context-specific behaviors more quickly.

\section{STRENGTHS AND LIMITATIONS}

The use of PALMS to detect outdoor time is relatively novel and the algorithm used by PALMS has been validated in one study (26), however further validation may be needed. We found a large proportion of the participant's time during school hours was spent outdoors. This may be a true finding but it may also be due to a problem with detecting outdoor time accurately at the schools included in this study. In many school buildings in Denmark, classrooms are situated along the outer walls with large windows. This combination may lead to a good satellite reception inside some classrooms, which could give some misclassification of indoor points being classified as outdoors. By definition, the proportion of time spent outdoors in contexts like playgrounds, urban green space, and active transport should have approximated $100 \%$ while we found averages ranging from 66.6 to $99.8 \%$. Manual inspection of the data confirmed that some epochs taking place outdoors were misclassified as indoors due to the SNR under heavy tree canopies or close to tall buildings presenting as low as 65. PALMS requires the SNR to be above 250 before classifying an epoch as outdoors. The PALMS classifications employ an SNR threshold, and reducing it would affect accuracy overall. Tree canopies are known to affect SNR so it is difficult to envisage a solution based on the GPS data alone. Post processing matched green space with the GPS data may help but cannot be automated. The prevalence of this problem is not known, but the impact of this misclassification will be an underestimation of the association between outdoor time and MVPA.
Almost all participants on two out of four schools had to be excluded as their GPS did not record the information needed to estimate outdoor time. This loss of data was due to malfunctioning Qstarz software not encountered during a pilot study. When detected, the open source software bt747 (bt747.org) was used instead, which eradicated the problem. When using novel technologies and devices, errors will inevitably happen, introducing possible systematic errors. The error happening in this study limited the number of participants that could be included, however it is not likely that this introduced a systematic error as the excluded participants did not differ from the included participants on important background characteristics.

This study focused on urban Danish children and as other studies have found differences in the urban/suburban/rural physical activity patterns $(15,54)$ the results may not be generalizable to more rural children. Also the children were selected based on their school attendance in four schools situated close to each other as they were part of a larger natural experiment evaluating changes to one specific local neighborhood. As such, further studies are needed to confirm the generalizability of the results. Data were only collected during early fall and late spring, where daylight and overall weather conditions were quite similar, and therefore it is also not known if the results are valid during the winter months (16).

This study is one of the first studies to describe the daily context-specific outdoor time and outdoor MVPA patterns among school-children using objective measures during weekdays. Future studies should also consider examining the weekend pattern as different patterns of physical activity have been described between week and weekend days (17). Also future studies should investigate if the association found between outdoor time and MVPA is consistent across subgroups. Age and gender interactions were present in some domains or subdomains but we chose not to stratify the data by subgroups due to sample size limitations. The sample size also cautions interpretation of the interactions presented in this paper and these results may not be generalizable to other populations. Future studies should further investigate age and gender interactions present in context-specific behavior and also investigate if other subgroups, e.g., overweight/obese, high/low socio-economic position, have a distinct context-specific pattern. However, researchers must consider the increased complexity this adds in presenting the data.

\section{CONCLUSION}

Different context-specific behaviors were found for gender and age, suggesting different strategies may be needed to promote physical activity among these groups. Studies using a combination of accelerometer and GPS devices are increasing in numbers as the need for context-specific physical activity patterns to inform effective health promotion is being acknowledged. Using novel technologies involves novel data processing methods and analytic strategies, and to promote a strong evidence base it is important that uniform methods are used, making it possible to compare results across studies and perhaps in the future to pool data to investigate country differences. This study proposed a domain based methodology expanded with a number of subdomains to assess the context-specific outdoor time and physical activity 
patterns among school-children and this methodology can easily be transferred to other populations.

\section{AUTHOR CONTRIBUTIONS}

Charlotte Demant Klinker conceived and coordinated the study, was responsible for its design, acquisition of data, data cleaning, statistical analyses, and drafted the manuscript. Jasper Schipperijn handled and processed the data, contributed to the acquisition of data, and data cleaning. Jens Troelsen conceived the study, and participated in its design. Jacqueline Kerr contributed with significant input to the outline of the manuscript and Annette Kjær Ersbøll came with statistical input. All authors revised the manuscript critically, and read and approved the final manuscript.

\section{ACKNOWLEDGMENTS}

The WCMC research team would like to thank all participating schools, students, teachers, principals, and school boards. We thank the Haraldsgade Urban renewal staff and the graduate students who assisted in the data collection, and others involved in the project. We thank Berhard Snized for invaluable help in building the database. Finally, we would like to thank Professor Bjørn Holstein for constructive feedback on earlier drafts of this manuscript. The When Cities Move Children study is part of the Centre for Intervention Research in Health Promotion and Disease Prevention, National Institute of Public Health, University of Southern Denmark. The Centre is funded by TrygFonden and the Danish Cancer Society. The WCMC study is funded by a 5-year donation from TrygFonden.

\section{REFERENCES}

1. McCurdy LE, Winterbottom KE, Mehta SS, Roberts JR. Using nature and outdoor activity to improve children's health. Curr Probl Pediatr Adolesc Health Care (2010) 40(5):102-17. doi:10.1016/j.cppeds.2010.02.003

2. Wen LM, Kite J, Merom D, Rissel C. Time spent playing outdoors after school and its relationship with independent mobility: a cross-sectional survey of children aged 10-12 years in Sydney, Australia. Int J Behav Nutr Phys Act (2009) 6:15. doi:10.1186/1479-5868-6-15

3. Bauman AE, Reis RS, Sallis JF, Wells JC, Loos RJ, Martin BW. Correlates of physical activity: why are some people physically active and others not? Lancet (2012) 380(9838):258-71. doi:10.1016/S0140-6736(12)60735-1

4. Sallis JF, Prochaska JJ, Taylor WC. A review of correlates of physical activity of children and adolescents. Med Sci Sports Exerc (2000) 32(5):963-75. doi:10.1097/00005768-200005000-00014

5. Ferreira I, van der Horst K, Wendel-Vos W, Kremers S, van Lenthe FJ, Brug J. Environmental correlates of physical activity in youth - a review and update. Obes Rev (2007) 8(2):129-54. doi:10.1111/j.1467-789X.2006.00264.x

6. Cleland V, Crawford D, Baur LA, Hume C, Timperio A, Salmon J. A prospective examination of children's time spent outdoors, objectively measured physical activity and overweight. Int J Obes (Lond) (2008) 32(11):1685-93. doi:10.1038/ijo.2008.171

7. World Health Organization. Global Recommendations on Physical Activity for Health. Geneva: WHO Press (2010).

8. Hallal PC, Andersen LB, Bull FC, Guthold R, Haskell W, Ekelund U. Global physical activity levels: surveillance progress, pitfalls, and prospects. Lancet (2012) 380(9838):247-57. doi:10.1016/S0140-6736(12)60646-1

9. Riddoch CJ, Bo Andersen L, Wedderkopp N, Harro M, Klasson-Heggebo L, Sardinha LB, et al. Physical activity levels and patterns of 9- and 15-yr-old European children. Med Sci Sports Exerc (2004) 36(1):86-92. doi:10.1249/01.MSS. 0000106174.43932 .92

10. Kristensen PL, Moller NC, Korsholm L, Wedderkopp N, Andersen LB, Froberg K. Tracking of objectively measured physical activity from childhood to adolescence: the European youth heart study. Scand J Med Sci Sports (2008) 18(2):171-8. doi:10.1111/j.1600-0838.2006.00622.x

11. Telama R. Tracking of physical activity from childhood to adulthood: a review. Obes Facts (2009) 2(3):187-95. doi:10.1159/000222244

12. Bauman AE, Sallis JF, Dzewaltowski DA, Owen N. Toward a better understanding of the influences on physical activity: the role of determinants, correlates, causal variables, mediators, moderators, and confounders. Am J Prev Med (2002) 23(2 Suppl):5-14. doi:10.1016/S0749-3797(02)00469-5

13. Sallis JF, Glanz K. The role of built environments in physical activity, eating, and obesity in childhood. Future Child (2006) 16(1):89-108. doi:10.1353/foc.2006. 0009

14. Dessing D, Pierik FH, Sterkenburg RP, van Dommelen P, Maas J, De Vries SI. Schoolyard physical activity of 6-11 year old children assessed by GPS and accelerometry. Int J Behav Nutr Phys Act (2013) 10:97. doi:10.1186/1479-586810-97

15. Rainham DG, Bates CJ, Blanchard CM, Dummer TJ, Kirk SF, Shearer CL. Spatial classification of youth physical activity patterns. Am J Prev Med (2012) 42(5):e87-96. doi:10.1016/j.amepre.2012.02.011

16. Oreskovic NM, Blossom J, Field AE, Chiang SR, Winickoff JP, Kleinman RE. Combining global positioning system and accelerometer data to determine the locations of physical activity in children. Geospat Health (2012) 6(2):263-72.

17. Maddison R, Jiang Y, Vander HS, Exeter D, Mhurchu CN, Dorey E. Describing patterns of physical activity in adolescents using global positioning systems and accelerometry. Pediatr Exerc Sci (2010) 22(3):392-407.

18. Kerr J, Duncan S, Schipperijn J. Using global positioning systems in health research: a practical approach to data collection and processing. Am J Prev Med (2011) 41(5):532-40. doi:10.1016/j.amepre.2011.07.017

19. Wheeler BW, Cooper AR, Page AS, Jago R. Greenspace and children's physical activity: a GPS/GIS analysis of the PEACH project. Prev Med (2010) 51(2):148-52. doi:10.1016/j.ypmed.2010.06.001

20. Lachowycz K, Jones AP, Page AS, Wheeler BW, Cooper AR. What can global positioning systems tell us about the contribution of different types of urban greenspace to children's physical activity? Health Place (2012) 18(3):586-94. doi:10.1016/j.healthplace.2012.01.006

21. Jones AP, Coombes EG, Griffin SJ, van Sluijs EM. Environmental supportiveness for physical activity in English schoolchildren: a study using global positioning systems. Int J Behav Nutr Phys Act (2009) 6:42. doi:10.1186/1479-5868-6-42

22. Krenn PJ, Titze S, Oja P, Jones A, Ogilvie D. Use of global positioning systems to study physical activity and the environment: a systematic review. Am J Prev Med (2011) 41(5):508-15. doi:10.1016/j.amepre.2011.06.046

23. Almanza E, Jerrett M, Dunton G, Seto E, Pentz MA. A study of community design, greenness, and physical activity in children using satellite, GPS and accelerometer data. Health Place (2012) 18(1):46-54. doi:10.1016/j.healthplace. 2011.09 .003

24. Rodriguez DA, Cho GH, Evenson KR, Conway TL, Cohen D, Ghosh-Dastidar B, et al. Out and about: association of the built environment with physical activity behaviors of adolescent females. Health Place (2012) 18(1):55-62. doi:10.1016/j.healthplace.2011.08.020

25. Cooper AR, Page AS, Wheeler BW, Hillsdon M, Griew P, Jago R. Patterns of GPS measured time outdoors after school and objective physical activity in English children: the PEACH project. Int J Behav Nutr Phys Act (2010) 7:31. doi:10.1186/1479-5868-7-31

26. Tandon PS, Saelens BE, Zhou C, Kerr J, Christakis DA. Indoor versus outdoor time in preschoolers at child care. Am J Prev Med (2013) 44(1):85-8. doi:10.1016/j.amepre.2012.09.052

27. The Physical Activity and Location Measurement (PALMS). Available from: http://ucsd-palms-project wikispaces com/ (2013).

28. Cho GH, Rodríguez DA, Evenson KR. Identifying walking trips using GPS data. Med Sci Sports Exerc (2011) 43(2):365-72. doi:10.1249/MSS.0b013e3181ebec3c

29. Kerr J, Normam G, Godbole S, Raab F, Demchak B, Patrick K. Validating GPS data with the PALMS system to detect different active transportation modes. Med Sci Sports Exerc (2012) 44:S2529.

30. Copenhagen Municipality. Foelles Boligsocial Helhedsplan for Haraldsgadekvarteret. Copenhagen (2007).

31. Santelli JS, Smith RA, Rosenfeld WD, DuRant RH, Dubler N, Morreale M, et al. Guidelines for adolescent health research. A position paper of the Society for Adolescent Medicine. J Adolesc Health (2003) 33(5):396-409. doi:10.1016/ S1054-139X(03)00290-8 
32. Sherar LB, Griew P, Esliger DW, Cooper AR, Ekelund U, Judge K, et al. International children's accelerometry database (ICAD): design and methods. BMC Public Health (2011) 11:485. doi:10.1186/1471-2458-11-485

33. Corder K, Ekelund U, Steele RM, Wareham NJ, Brage S. Assessment of physical activity in youth. J Appl Physiol (2008) 105(3):977-87. doi:10.1152/japplphysiol. 00094.2008

34. Rowlands AV. Accelerometer assessment of physical activity in children: an update. Pediatr Exerc Sci (2007) 19(3):252-66.

35. Duncan S, Stewart TI, Oliver M, Mavoa S, Macrae D, Badland HM, et al. Portable global positioning system receivers: static validity and environmental conditions. Am J Prev Med (2013) 44(2):e19-29. doi:10.1016/j.amepre.2012.10.013

36. Pedersen CB. The Danish Civil Registration System. Scand J Public Health (2011) 39(7 Suppl):22-5. doi:10.1177/1403494810387965

37. Petersson F, Baadsgaard M, Thygesen LC. Danish registers on personal labour market affiliation. Scand J Public Health (2011) 39(7 Suppl):95-8. doi:10.1177/ 1403494811408483

38. Cole TJ, Bellizzi MC, Flegal KM, Dietz WH. Establishing a standard definition for child overweight and obesity worldwide: international survey. BMJ (2000) 320(7244):1240-3. doi:10.1136/bmj.320.7244.1240

39. Evenson KR, Catellier DJ, Gill K, Ondrak KS, McMurray RG. Calibration of two objective measures of physical activity for children. J Sports Sci (2008) 26(14):1557-65. doi:10.1080/02640410802334196

40. Trost SG, Loprinzi PD, Moore R, Pfeiffer KA. Comparison of accelerometer cut points for predicting activity intensity in youth. Med Sci Sports Exerc (2011) 43(7):1360-8. doi:10.1249/MSS.0b013e318206476e

41. Troiano RP, Berrigan D, Dodd KW, Masse LC, Tilert T, McDowell M. Physical activity in the United States measured by accelerometer. Med Sci Sports Exerc (2008) 40(1):181-8. doi:10.1249/mss.0b013e31815a51b3

42. Guinhouya BC, Lemdani M, Vilhelm C, Hubert H, Apete GK, Durocher A. How school time physical activity is the "big one" for daily activity among schoolchildren: a semi-experimental approach. J Phys Act Health (2009) 6(4):510-9.

43. Pratt M, Macera CA, Sallis JF, O’Donnell M, Frank LD. Economic interventions to promote physical activity: application of the SLOTH model. Am J Prev Med (2004) 27(3 Suppl):136-45. doi:10.1016/j.amepre.2004.06.015

44. Dencker M, Svensson J, El-Naaman B, Bugge A, Andersen LB. Importance of epoch length and registration time on accelerometer measurements in younger children. J Sports Med Phys Fitness (2012) 52(2):115-21.

45. Niclasen B, Petzold M, Schnohr CW. The association between high recreational physical activity and physical activity as a part of daily living in adolescents and availability of local indoor sports facilities and sports clubs. Scand J Public Health (2012) 40(7):614-20. doi:10.1177/1403494812459815

46. Quigg R, Gray A, Reeder AI, Holt A, Waters DL. Using accelerometers and GPS units to identify the proportion of daily physical activity located in parks with playgrounds in New Zealand children. Prev Med (2010) 50(5-6):235-40. doi:10.1016/j.ypmed.2010.02.002
47. Reed JA, Hooker SP. Where are youth physically active? A descriptive examination of 45 parks in a southeastern community. Child Obes (2012) 8(2):124-31. doi:10.1089/chi.2011.0040

48. Griew P, Page A, Thomas S, Hillsdon M, Cooper AR. The school effect on children's school time physical activity: the PEACH project. Prev Med (2010) 51(3-4):282-6. doi:10.1016/j.ypmed.2010.06.009

49. Bringolf-Isler B, Grize L, Mader U, Ruch N, Sennhauser FH, Braun-Fahrlander C. Built environment, parents' perception, and children's vigorous outdoor play. Prev Med (2010) 50(5-6):251-6. doi:10.1016/j.ypmed.2010.03.008

50. Yildirim M, Schoeni A, Singh AS, Altenburg T, Brug J, De Bourdeaudhuij I, et al. Daily variations in weather and the relationship with physical activity and sedentary time in European 10-12yr olds: the ENERGY-Project. J Phys Act Health (2014) 11(2):419-25. doi:10.1123/jpah.2012-0102

51. Aarts MJ, De Vries SI, van Oers HA, Schuit AJ. Outdoor play among children in relation to neighborhood characteristics: a cross-sectional neighborhood observation study. Int J Behav Nutr Phys Act (2012) 9(1):98. doi:10.1186/1479-58689-98

52. Toftager M, Christiansen LB, Kristensen PL, Troelsen J. SPACE for physical activity - a multicomponent intervention study: study design and baseline findings from a cluster randomized controlled trial. BMC Public Health (2011) 11:777. doi:10.1186/1471-2458-11-777

53. International Physical Activity and the Environment Network (IPEN). Available from: http://www ipenproject org/IPEN_adolescent html (2013).

54. Fairclough SJ, Beighle A, Erwin H, Ridgers ND. School day segmented physical activity patterns of high and low active children. BMC Public Health (2012) 12(1):406. doi:10.1186/1471-2458-12-406

Conflict of Interest Statement: The authors declare that the research was conducted in the absence of any commercial or financial relationships that could be construed as a potential conflict of interest.

Received: 13 November 2013; accepted: 21 February 2014; published online: 11 March 2014.

Citation: Klinker CD, Schipperijn J, Kerr J, Ersbøll AK and Troelsen J (2014) Contextspecific outdoor time and physical activity among school-children across gender and age: using accelerometers and GPS to advance methods. Front. Public Health 2:20. doi: 10.3389/fpubh.2014.00020

This article was submitted to Public Health Education and Promotion, a section of the journal Frontiers in Public Health.

Copyright (C) 2014 Klinker, Schipperijn, Kerr, Ersbøll and Troelsen. This is an openaccess article distributed under the terms of the Creative Commons Attribution License (CC BY). The use, distribution or reproduction in other forums is permitted, provided the original author(s) or licensor are credited and that the original publication in this journal is cited, in accordance with accepted academic practice. No use, distribution or reproduction is permitted which does not comply with these terms. 